\title{
Hybrid-Order Network Consensus for Distributed Multi-agent Systems
}

\author{
Guangqiang Xie \\ Junyu Chen \\ Yang Li \\ College of Computer Science, \\ Guangdong University of Technology, Mail Stop: 510006, \\ No.100 Waihuanxi Road, Guangzhou HEMC, Guangdong
}

\author{
XIEGQ@GDUT.EDU.CN \\ NNCHENJUNYU@QQ.COM \\ LIYANG@GDUT.EDU.CN
}

\begin{abstract}
As an important field of Distributed artificial intelligence (DAI), multi-agent systems (MASs) have attracted the attention of extensive research scholars. Consensus as an important issue in MAS, good progress has been made in studying the consensus control of MAS, but there are some problems remained largely unaddressed which cause the MAS to lose some useful network structure information. First, multi-agent consensus protocol usually proceeds over the low-order structure by only considering the direct edges between agents, but ignores the higher-order structure of the whole topology network. Second, the existing work assumes all the edges in a topology network have the same weight without exploring the potential diversity of the connections. In this way, multi-agent systems fail to enforce consensus, resulting in fragmentation into multiple clusters. To address the above issues, this paper proposes a Motif-Aware Weighted Multi-agent System (MWMS) method for consensus control. We focus more on triangle motif in the network, but it can be extended to other kinds of motifs as well. First, a novel weighted network is used which is the combination of the edge-based lower-order structure and the motif-based higher-order structure, i.e., hybrid-order structure. Subsequently, by simultaneously considering the quantity and the quality of the connections in the network, a novel consensus framework for MAS is designed to update agents. Then, two baseline consensus algorithms are used in MWMS. In our experiments, we use ten topologies of different shapes, densities and ranges to comprehensively analyze the performance of our proposed algorithms. The simulation results show that the hybrid higher-order network can effectively enhance the consensus of the multi-agent system in different network topologies.
\end{abstract}

\section{Introduction}

Distributed Artificial Intelligence (DAI) mainly studies how logically or physically dispersed intelligent systems solve problems in parallel and in cooperation with each other. DAI can be divided into two basic research areas: Distributed Problem Solving (DPS) and Multiagent Systems (MAS). As an important field of DAI, MASs have attracted an increasing amount of attention from experts in computer science ${ }^{1}$, control theory (Dobbe, FridovichKeil, \& Tomlin, 2017), cooperative ${ }^{2}$, engineering ${ }^{3}$, reinforcement learning (Lin, Adams, \&

1. (Jiang \& Lu, 2018; Han \& Gmytrasiewicz, 2018; Song, Ren, Sadigh, \& Ermon, 2018; Ephrati \& Rosenschein, 1996)

2. (da Silva, Wu, Dai, \& Lin, 2016; Le \& Plaku, 2019; Dutta, Jennings, \& Moreau, 2005)

3. (Wang, Wei, Fan, Liu, \& Huang, 2019; Le \& Plaku, 2018; Kraus, 1997) 
Beling, 2019; Wai, Yang, Wang, \& Hong, 2018), etc. MASs should consider uncertainty and limited communication in order to generate robust solutions of high-quality (Amato, 2018; Bloembergen, Tuyls, Hennes, \& Kaisers, 2015). Since each agent has limited sensing, computing, storage and motion control capability, it is necessary for agent to cooperate with its neighbors in a distributed method to make the system achieve convergence in finite time (Ren \& Beard, 2005). In complex networks, multi-agent systems are mainly used to study the influence of algebraic connectivity on systems (Olfati-Saber, 2005).

MAS mainly studies the coordination of agent behavior, how to coordinate their knowledge, goals, skills and mutual planning between a group of autonomous agents to take action or solve problems. The agent in MAS can have a global goal or an independent goal related to itself. They must follow the coordination procedure of the behavior between agents. The basic difficulty to achieve system consensus is not to use centralized control. The basic problem that DAI researchers are trying to solve is to achieve global or local consensus only through local control (Bond, 1988). Olfati-Saber et al. (2007) provide a theoretical framework for analysis of consensus algorithms for networked multi-agent systems with fixed or unfixed topology and directed information flow. On this basis, many efforts have been made in developing decentralized consensus algorithms, such as hybrid multi-agent system (Zheng, Ma, \& Wang, 2018; Nebel, Bolander, Engesser, \& Mattmüller, 2019), coordination problem of multi-agents (Bhargava, 2019; Li, Yang, Cai, Yang, \& Wang, 2019), consensus of multi-agent systems (Xu \& He, 2018; Motsch \& Tadmor, 2014).

Network stands for the information interaction between agents and is the basis of distributed computing. However, the above existing methods only consider the design of the consensus protocol with edge-based connectivity pattern but ignore the higher-order connectivity structure which can reveal richer information between nodes, such as the number of common neighbors. One-hop-based consensus may lose some important but hard-to-discover connections. If this information can be fully used, multi-agent distributed computation over networks will have greater advantages. These problems can be addressed by motif which is a representative higher-order structure occurring in complex networks at numbers that are significantly higher than those in randomized networks preserving the same degrees of nodes ${ }^{4}$. In social network, the triangle motif composed of three vertices and three edges is a crucial basis for building social relations ${ }^{5}$. For example, Li et al. use motif to address the problem of low-order network structure on individual nodes and edges in the network community detection (Li, Huang, Wang, \& Lai, 2019). At present, motifs are mostly used for analyzing network structure in biology (Kim \& Choi, 2009), engineering (Milo, ShenOrr, Itzkovitz, Kashtan, Chklovskii, \& Alon, 2002), social media (Coletto, Garimella, Gionis, \& Lucchese, 2017) and statistical relational learning (Das, Dhami, Kunapuli, Kersting, \& Natarajan, 2019). However, there is still a lack of work studying and leveraging motif in MASs. According to our experiments, the introduction of motif to consensus protocols in MASs can achieve better results.

In this paper, we for the first time integrate motif into MASs and propose a Motif-Aware Weighted Multi-agent System (MWMS) framework for consensus control. In the previous consensus control methods, the number of the topological edges formed by agents and all

4. (Zhou, Zhang, Yildirim, Alcorn, Tong, Davulcu, \& He, 2017; Newman, 2006; Li, Cai, Wang, Liang, \& Zheng, 2019; Huang, Wang, \& Chao, 2019)

5. (Li, Dau, Puleo, \& Milenkovic, 2017; Li, Huang, Wang, Huang, \& Lai, 2018; Song, Zou, \& Liu, 2016) 
its neighbors is usually used as an important measure of neighborhood weight. Although this approach can make the system stable, it may split all agents into multiple clusters. The main reason is that some useful information is ignored if simply using the lower-order connectivity edges as weight coefficient. Due to the characteristics of motif, we can consider the higher-order structure as the reference for neighborhood weight. We design a hybrid consensus framework model by taking into account both of lower-order connectivity pattern and higher-order connectivity pattern, which is different from the traditional consensus algorithms. In our consensus algorithm framework, the triangle motifs containing agents are used as one of the sources of the edge weight coefficient, which is not just 0 and 1 . The reason is that the number of triangle motifs depicts the relationships between itself and neighbors, neighbors and neighbors to a certain extent. We can regard the traditional algorithm as a special case of our algorithm in this paper. In our experiments, we compare the traditional algorithms with the proposed algorithms under the same initial topology network. The results show that the convergence effect of the proposed MWMS framework for MASs is better, i.e., the splitting of the system can be effectively reduced by introducing motif into the control function.

The rest of this paper is organized as follows. In section 2, some definitions of symbols and necessary knowledge are given to facilitate the following model description. Section 3 contains the construction of the MWMS framework and its application in multi-agent consensus. In section 4, a large number of simulation results are given. At the same time, we analyze the relationship between parameters and topological network. Section 5 summarizes the contributions made by this paper and discusses future research directions.

\section{Preliminary Knowledge and Problem Statement}

In this section, we will introduce some symbols related to multi-agents and the definition of motif. Then, the problem we solve in this paper will be explained.

\subsection{Graph and Matrix Theory}

We describe a multi-agent system with $n$ agents as an undirected graph $G=\{V, E, A\}$. $V$ is the vertex set of graph $G$ with each vertex being an agent. $E$ is the edge set of graph $G$, which satisfies $E \subset V \times V$. One edge $(i, j) \in E$ of graph $G$ indicates that a pair of agents $i$ and $j$ are neighbors, and they can exchange information with each other. $A=\left(a_{i j}\right)_{n \times n}$ is an adjacency matrix of graph $G$. The elements of $A$ are related to edges, i.e., $a_{i j}=1 \Leftrightarrow(i, j) \in E$. The neighbor set of agent $i$ is represented as

$$
\mathcal{N}_{i}=\{j \in V \mid(i, j) \in E\}
$$

This paper assumes that there is no self-loop in graph $G$, i.e., the main diagonal elements $a_{i i}=0, \forall i$. If any two agents in graph $G$ are neighbors, then graph $G$ is called a complete graph. The path from agent $i$ to another agent $j$ is defined as a sequence of different agents, where $i$ is the start and $j$ is the end, and each agent in the sequence is a neighbor with the agent next to it. If there is a path from agent $i$ to agent $j$, then the two agents are said to be connected. If any pair of agents in MAS are connected, $G$ is called a connected graph. The degree matrix $D$ of graph $G$ is defined as $D=\operatorname{diag}\left\{d_{i}\right\}$, where $d_{i}=\sum_{j \in \mathcal{N}_{i}} a_{i j}$ is the degree of 


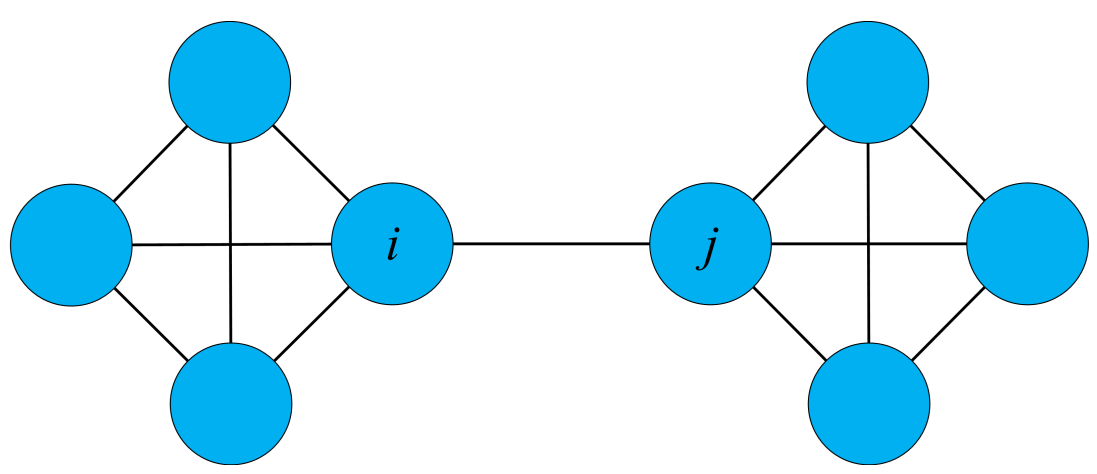

Figure 1: An example of multi-agent systems with limited sensing range prone to splitting.

vertex $i$, i.e., $\left|\mathcal{N}_{i}\right|$ is the number of neighbors $\mathcal{N}_{i}$. Accordingly, Laplacian matrix $L$ of graph $G$ is defined as $L=D-A$.

\subsection{Traditional Discrete Consensus Protocol}

Consider a multi-agent system consisting of $n$ agents. We use $G(t)=\{V, E(t), A(t)\}$ to represent the graph structure at time $t$. The state value of agent $i$ at time $t$ is represented by $x_{i}(t)$. In this paper, it is assumed that agents can only communicate with neighbor agents in a limited sensing range $r_{c}$. The neighbor set of agent $i$ is

$$
\mathcal{N}_{i}(t)=\left\{j:\left\|x_{j}(t)-x_{i}(t)\right\| \leq r_{c}\right\} .
$$

where $\|\bullet\|$ represents the second normal form of vector $\bullet$.

Olfati-Saber et al. (2007) proposed a discrete distributed consensus algorithm. The algorithm can be expressed as

$$
x_{i}(t+1)=x_{i}(t)+\varepsilon \sum_{j \in \mathcal{N}_{i}(t)} a_{i j}(t)\left(x_{j}(t)-x_{i}(t)\right)
$$

where $\varepsilon>0$ is the learning rate. In general, $\varepsilon \in\left(0, \frac{1}{n}\right]$. And $a_{i j}(t)$ is binary, i.e., if $j \in \mathcal{N}_{i}(t)$, then $a_{i j}(t)=1$, otherwise $a_{i j}(t)=0$. The discrete-time collective dynamics of the network under this algorithm can be written as

$$
X(t+1)=P(t) X(t)
$$

where $P(t)=(I-\varepsilon L(t))$ with $I$ being the identity matrix and $L(t)$ being the Laplace matrix at time $t . L(t)$ is defined as

$$
L_{i j}(t)= \begin{cases}\left|\mathcal{N}_{i}(t)\right|, & i=j \\ -1, & j \in \mathcal{N}_{i}(t) \\ 0, & j \notin \mathcal{N}_{i}(t)\end{cases}
$$

Another popular algorithm is proposed by Jadbabaie, Lin and Morse (2003), also discussed by Olfati-Saber et al. (2007), which is the following discrete-time consensus algorithm for undirected networks. 


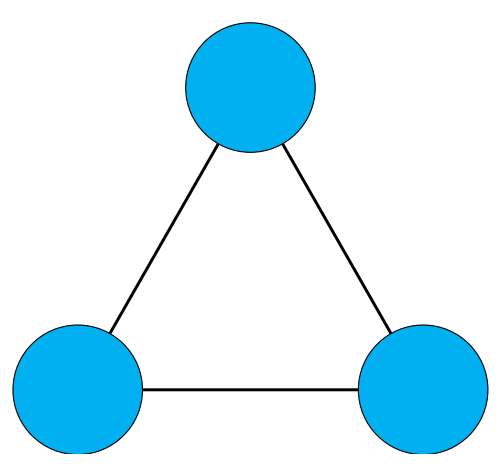

(a) $\Upsilon(3,3)$

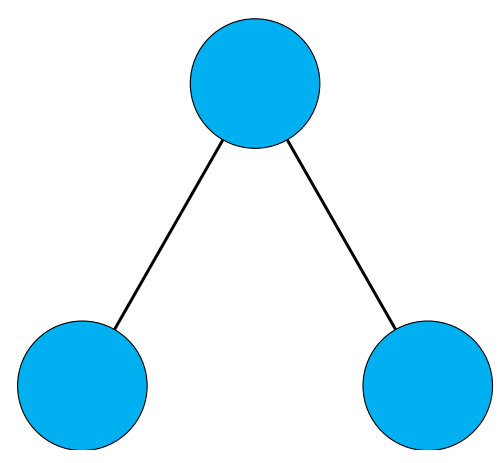

(b) $\Upsilon(3,2)$

Figure 2: Illustration of motifs: The undirected motif with 3 nodes and 2 edges (resp. 3 nodes and 2 edges) is denoted by $\Upsilon(3,3)$ (resp. $\Upsilon(3,2)$ ).

$$
x_{i}(t+1)=\frac{1}{1+\left|\mathcal{N}_{i}(t)\right|}\left(x_{i}(t)+\sum_{j \in \mathcal{N}_{i}(t)} x_{j}(t)\right)
$$

which can be written as

$$
X(t+1)=(I+D(t))^{-1}(I+A(t)) X(t)
$$

Topological connectivity is closely related to the distance (state) between agents: when the distance between agents is less than a certain threshold value, they are considered to be communication connected (correspondingly, when the state value is less than a certain threshold value, they are considered to be similar and can be used for mutual reference information); otherwise, there is no connection, i.e., time-varying dynamic network ${ }^{6}$. In this case, the theory proofs and effect of traditional protocol will become inapplicable. In short, these methods are not effective in multi-agent systems with limited sensing range. For example, as shown in Figure 1, the weight of each edge is 1. According to the traditional consensus algorithm, agent $i$ will converge to the left; otherwise, agent $j$ will converge to the right. The edge $(i, j)$ is broken, and the system finally forms two clusters. The reason is that edges $(i, j)$ are fragile, they do not have common neighbors, and this information cannot be reflected in low-level network structures. Therefore, we need to consider higher-order features of the network to enhance the consensus, e.g., motifs.

\subsection{Motif}

The most common higher-order structures are small network subgraphs, which we refer to as network motifs. Network motifs are considered as the building blocks for complex networks. Given a graph $G$, a network motif in $G$ is a subgraph $g$ of $G$, such that $g$ appears much more frequently in $G$ than in random graphs whose degree distributions are similar to that of $G$. To some extent, network motif reveals a deeper relationship structure in $G$. An

6. (Cao, Ren, Casbeer, \& Schumacher, 2016; Dong, 2010; Ma, Zheng, \& Wang, 2015; Zhang, Wang, \& Zhang, 2015; Cao \& Ren, 2009) 

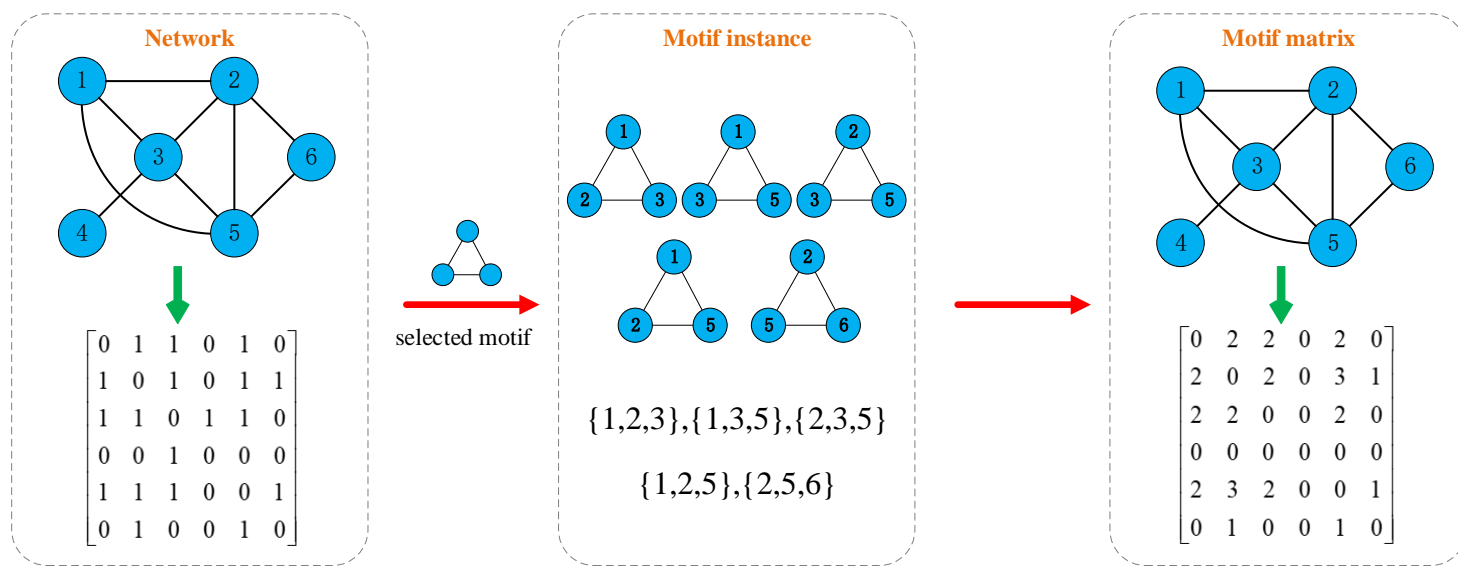

Figure 3: The construction of the motif adjacency matrix based on $\Upsilon(3,3)$.

adjacent matrix $A$ represents the lower-order structure of graph $G$, while a motif matrix $M$ represents the higher order structure of graph $G$. The network motif is formally defined as follows (Milo et al., 2002).

Definition 1. Motif is a dense subgraph occurring in complex networks at numbers that are significantly higher than those in randomized networks preserving the same degree of nodes. It is denoted as $g=\left\{V_{g}, E_{g}\right\}$ where $V_{g}$ and $E_{g}$ denote the node set consisting of $m$ nodes and edge set consisting of $l$ edges in the motif $g$ respectively, with $l$ being between $m-1$ ( $a$ line motif) and $\frac{m(m-1)}{2}$ (a clique motif).

We can analyze different motifs discovered from various types of networks. Among them, the 3-node motifs and 4-node motifs composed of 3 nodes and 4 nodes are the most widely studied respectively, due to their wide appearance in diverse networks as building blocks. Following the conventional setting ${ }^{7}$, we focus on triangle motifs in an undirected network of multi-agents. But our technique can be extended to other types of motifs. For clarity, we denote $\Upsilon(p, q)$ as the undirected motif with $p$ nodes and $q$ edges. For example, the two motifs in Figure 2 can be denoted as $\Upsilon(3,3)$ and $\Upsilon(3,2)$ respectively. They can effectively reflect intimate relationship and the connectivity quality between two agents.

\subsection{Problem Statement}

In this paper, we focus on optimizing the protocol to enhance the consensus of the MAS, rather than the topology control of the network. Therefore, we mainly solve two inevitable problems in the baseline protocol:

1. Multi-agent consensus algorithm usually proceeds over the lower-order structure by only considering the direct edges between agents, but ignores the higher-order structure of the whole topology network.

7. (Newman, 2001; Newman \& Park, 2003; Huang, Wang, \& Chao, 2018; Wen, Gao, Fu, Zhang, \& Xia, 2019) 
2. All the edges in a topology network have the same weight without exploring the potential diversity of the connections.

The above two problems cause the communication network of the traditional consensus protocol to be simple, and they cannot accurately reflect the richer connection information between the two agents. The effective use of these informations can contribute to the consensus of the MAS. Based on the knowledge of multi-agent systems and network motifs, we simultaneously consider the lower-order structure and higher-order structure of network, and design a more general consensus algorithm which enhances the consensus of multi-agent systems.

\section{The Proposed Method}

In this section, we describe in detail the proposed Motif-Aware Weighted Multi-agent System (MWMS) framework for consensus.

\subsection{Motif-Based Hybrid-Order Structure}

Given a network $G(t)=\{V, E(t), A(t)\}$ of $n$ agents at time $t$. Suppose the network is initially connected. There are two subproblems need to be solved. The first subproblem is to find the recurring patterns, i.e., motifs, which are statistically overrepresented compared with those in the corresponding random networks. Many efforts have been made in addressing this task, such as Kavosh (2009), FANMOD (Wernicke \& Rasche, 2006) and GPU-based solution (Lin, Xiao, Xie, \& Li, 2017).

As for the second subproblem, we use motif to construct the higher-order motif adjacency matrix $M(t)$, whose elements represent the number of co-existing of the corresponding two agents in an instance of the identified motif.

$$
M_{i j}(t)=N_{i j}^{W}(t)
$$

where $N_{i j}^{W}(t)$ represents the number of the identified motif instances that contain agent $i$ and $j$. As shown in Figure 3, for example, there are totally five identified motif instances, and three of them contain agent 2 and 5 so that $M_{25}(t)=M_{52}(t)=3$. It's worth noting that different networks may have different motifs and may give different interpretations to the identified motifs according to the characteristics of the network. In other words, different motifs can model different kinds of relations in the network. In this example, we just take the triangle motif motif for illustration purpose. Once the motif is identified, we can infer the prevalent types of relations in the network by giving interpretations to the identified motif. Hence, the structural characteristics can be uncovered and the second subproblem is solved.

The motif matrix can express more abundant relationships between any two points. Each edge is no longer weighted by 1 , but by a different positive integer representing the higher-order connectivity pattern. In particular, the larger the element value of the motif matrix is, the closer the relationship between the corresponding two agents is. Motif matrix gives us an intuitive understanding of how close agents are to each other. Therefore, we propose a weighted hybrid matrix as follows:

$$
W(t)=(1-\alpha) A(t)+\alpha M(t)
$$


where $A(t)$ is the adjacency matrix and $M(t)$ is the motif adjacency matrix at time $t$. $\alpha \in[0,1]$ is the trade-off parameter which balances the importance of two matrices. In this way, the aforementioned problems suffered by multi-agent system are solved.

\subsection{The Motif-Aware Weighted Multi-agent System}

Before using motif adjacency matrix to improve the existing consensus algorithm, we need to understand the mechanism of hybrid-order structures.

In Eq. 9, it is shown that the value of each entry of the matrix represents the stability between agents. In the case of triangle motif, the larger the $W_{i j}(t)$ value is, the more stable the relationship between agent $i$ and $j$ is at time $t$. Motsch et al. (2014) hold that "heterophily", the tendency to bond more with those who are different rather than with those who are similar, plays a decisive role in the process of clustering. In order to enhance the consensus of the system, for each agent, the neighbor with relatively unstable structure is bound to have greater weights. On the contrary, those with stable structure can relax the constraint appropriately. As a result, the reciprocal is used to illustrate this idea. To facilitate our work, we need to define some notations. Let $W^{r}$ denote a matrix whose elements are reciprocal to the corresponding element of the adjacency matrix $W$. That is

$$
W_{i j}^{r}(t)=\left\{\begin{array}{l}
\frac{1}{W_{i j}(t)}, \quad W_{i j}(t) \neq 0 \\
0
\end{array}\right.
$$

Likewise, let

$$
D^{r}(t)=\operatorname{diag}\left\{d_{i}^{r}(t)\right\}
$$

where $d_{i}^{r}(t)=\sum_{j \in \mathcal{N}_{i}(t)} W_{i j}^{r}(t)$. In fact, $D^{r}(t)$ is similar to a weighted degree matrix, which is related to $W(t)$.

So far, we have explored the significance of element value of hybrid matrix in multi-agent system, and made corresponding adjustment according to its value.

\subsection{Traditional Consensus Algorithms based on Motif-Aware Weighted Multi-agent System}

We incorporate motif into the classical consensus algorithm, and show that the traditional algorithms are special cases of our algorithms. Based on Motif-Aware Weighted Multi-agent framework, Eq. 3 can be written as

$$
x_{i}(t+1)=x_{i}(t)+\varepsilon \sum_{j \in \mathcal{N}_{i}(t)}\left|\mathcal{N}_{i}(t)\right| \frac{W_{i j}^{r}(t)}{D_{i i}^{r}(t)}\left(x_{j}(t)-x_{i}(t)\right)
$$

where $\varepsilon>0$ denotes the learning rate, $\left|\mathcal{N}_{i}(t)\right|$ stands for the number of neighbors of agent $i$ at time $t$. In fact, compared with the traditional algorithm, $a_{i j}(t)$ in MWMS is not a binary value. At the same time, in order to compare with the same benchmark, both equations satisfy $\sum_{j \in \mathcal{N}_{i}(t)} a_{i j}(t)=\left|\mathcal{N}_{i}(t)\right|$. If $\alpha$ in Eq. (9) is equal to zero, which means only the lowerorder connectivity pattern is considered without taking motif structure into account. The 


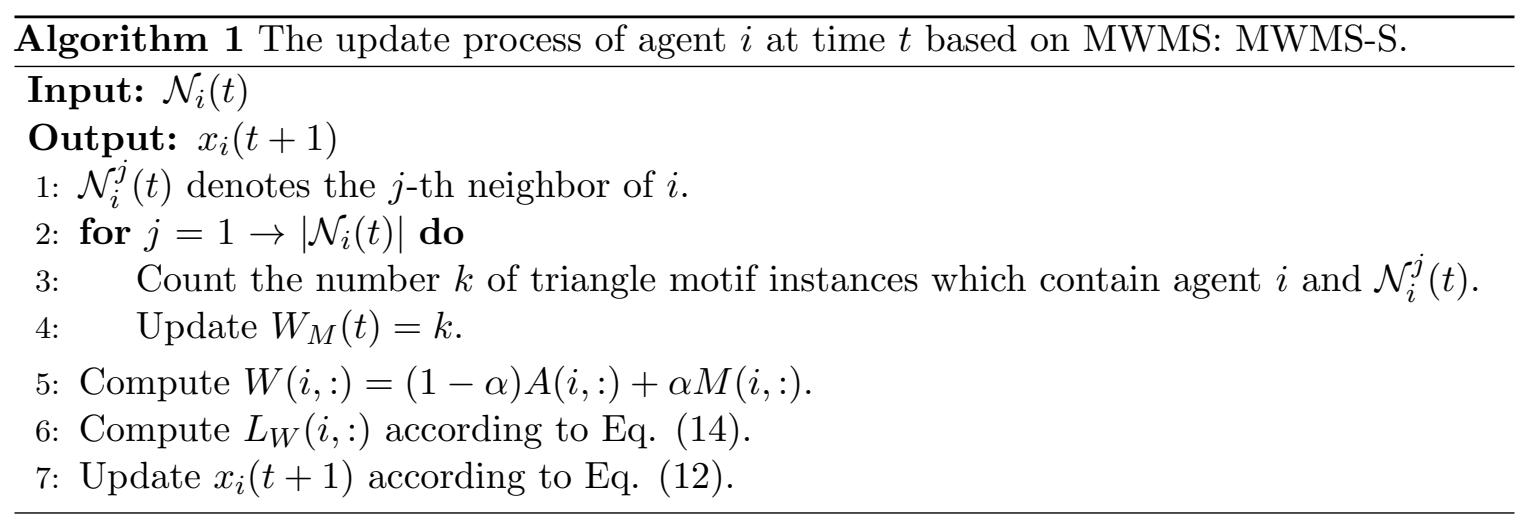

traditional algorithm and the MWMS algorithm are equivalent constitutionally. Similarly, the matrix form of Eq. (12) can be written as

$$
X(t+1)=P_{W}(t) X(t)
$$

with $P_{W}(t)=(I-\varepsilon \widehat{L}(t))$ where $\widehat{L}(t)$ is a matrix having the similar form as Laplace matrix $L$. And the elements of $\widehat{L}(t)$ are

$$
\widehat{L}_{i j}(t)= \begin{cases}\left|\mathcal{N}_{i}(t)\right|, & j=i \\ -\left|\mathcal{N}_{i}(t)\right| \cdot \frac{W_{i j}^{r}(t)}{D_{i i}^{r}(t)}, & j \in \mathcal{N}_{i}(t) \\ 0 & \end{cases}
$$

For clarity, $\widehat{L}(t)$ can be written as

$$
\widehat{L}(t)=D(t)-D(t) \cdot\left(D^{r}(t)\right)^{-1} \cdot W^{r}(t)
$$

where $D(t)$ is the degree matrix of graph $G(t)$ at time $t$. For clarity, Algorithm 1 summarizes the updated process of agent $i$ at time $\mathrm{t}$ based on MWMS.

Secondly, we apply the hybrid-order motif matrix to Eq. 6. It can be expressed as

$$
x_{i}(t+1)=\sum_{j \in \mathcal{N}_{i}(t) \cup\{i\}} b_{i j}(t) x_{j}(t)
$$

where $b_{i j}(t)$ is defined as

$$
b_{i j}(t)= \begin{cases}\frac{W_{i j}^{r}(t)}{D_{i i}^{r}(t)+1}, & j \in \mathcal{N}_{i}(t) \\ \frac{1}{D_{i i}^{r}(t)+1}, & j=i \\ 0 & \end{cases}
$$

Compare with Eq. 6 whose $b_{i j}(t)=\frac{1}{1+\left|\mathcal{N}_{i}(t)\right|}$, Eq. 17 also satisfies $\sum_{\mathcal{N}_{i}(t) \cup\{i\}} b_{i j}(t)=\left|\mathcal{N}_{i}(t)\right|+$ 1. The difference is that each neighbor of agent $i$ is given different weights, instead of equal 


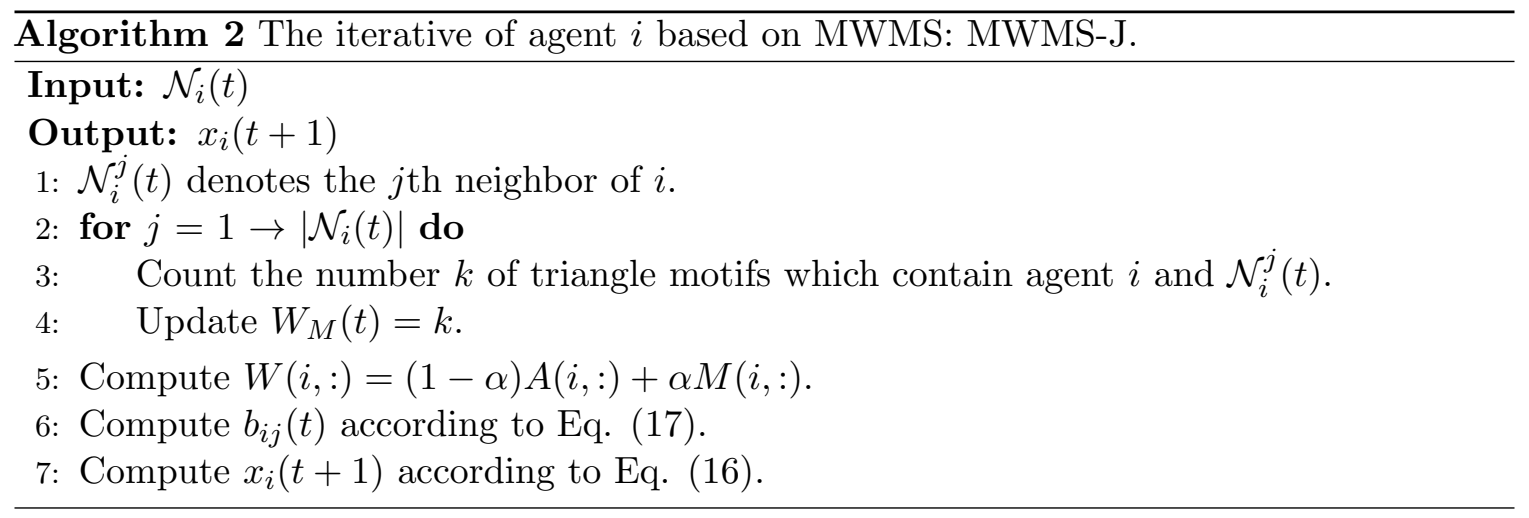

weights, due to the consideration of adding motif matrix which can reveal the deep relations that the adjacency matrix cannot. And then Eq. 16 can be written as

$$
X(t+1)=\left(I+D^{r}(t)\right)^{-1}\left(I+W^{r}(t)\right) X(t) .
$$

When $\alpha$ is equal to zero in Eq. 9, the consensus algorithm mentioned above is equivalent to Eq. 7. Meanwhile, we can find that $\alpha$ is a parameter adjusting its weight. The bigger $\alpha$ is, the bigger $b_{i i}(t)$ has to be. The process is described by Algorithm 2.

For clarity, Algorithm 1 is called MWMS-S and Algorithm 2 is called MWMS-J. In conclusion, MWMS-S and MWMS-J are based on three node motif, so the time complexity for each agent is $\mathcal{O}\left(\frac{n^{2}}{2}\right)$ at time $t$, where $n$ is the number of neighbors of agent $i$. A more complex motif matrix would be better calculated if the agent had more computational power. It is worth noting that, because of the addition of motif, the original symmetric adjacency matrix becomes asymmetrical motif matrix, it is difficult to prove the convergence. So we prove its convergence through a lot of simulation experiments.

\section{Experiments}

The experiment is mainly divided into two sets. In the first set of experiments, we explored the effects of ten different initial topologies on the results of the algorithm. In the second set of experiments, we focused on the square area and explored the effect of the initial topology of different densities and ranges on the algorithm results. In our experiments, 11 different values of $\alpha$ are tested, that is $\alpha \in\{0,0.1,0.2,0.3,0.4,0.5,0.6,0.7,0.8,0.9,1\}$. In addition, $r_{c}=1$ and $\varepsilon=\frac{1}{n}$. Due to the different final effects among the algorithms, the traditional algorithm has been split into multiple clusters after several iterations to achieve stability. Under the MWMS framework, the system can gradually reach consensus or just split into several clusters, and the time to reach stability of the system must be longer than the traditional method. In this case, the comparison of convergence rates is meaningless because the results of convergence are different. Therefore, the number of clusters when multi-agent system is stable is utilized to evaluate the performance of consensus algorithm for evaluation. Smaller cluster number indicates better results. 


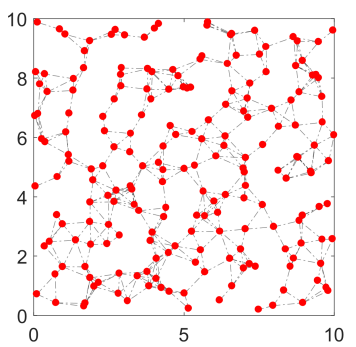

(a) Top-2: 200 agents

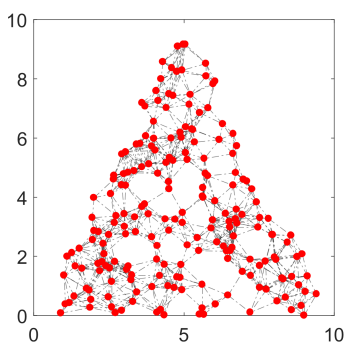

(e) Top-5: 200 agents

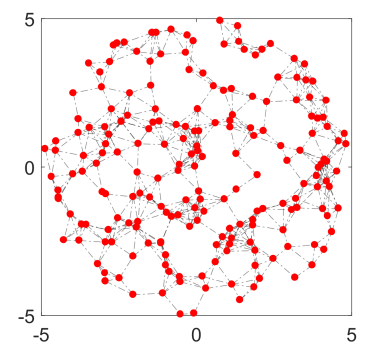

(b) Top-1: 200 agents

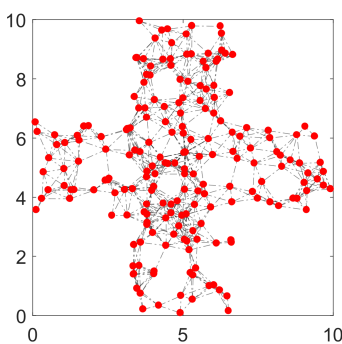

(f) Top-6: 200 agents

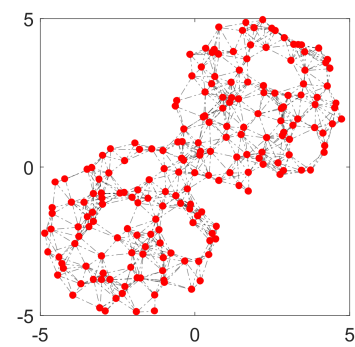

(c) Top-3: 200 agents

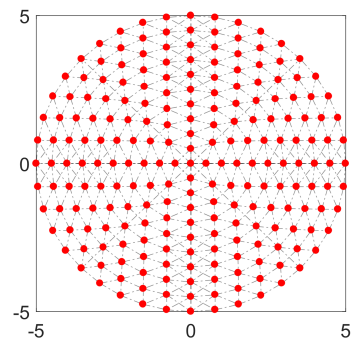

(g) Top-7: 221 agents

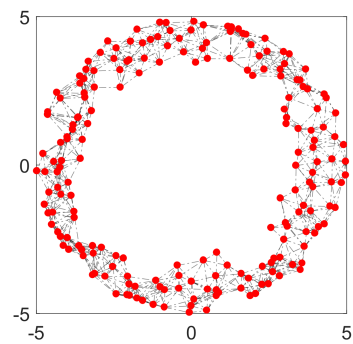

(d) Top-4: 200 agents

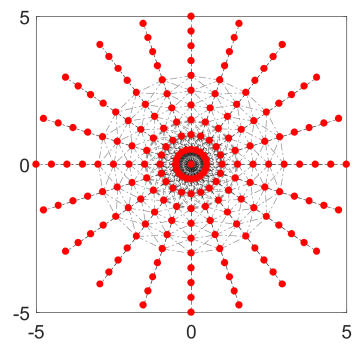

(h) Top-8: 217 agents

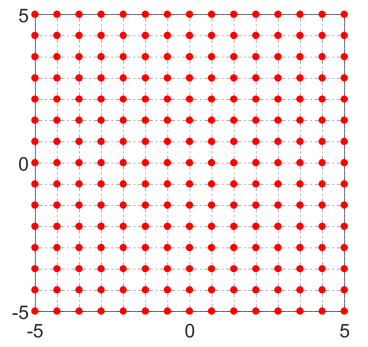

(i) Top-9: 225 agents

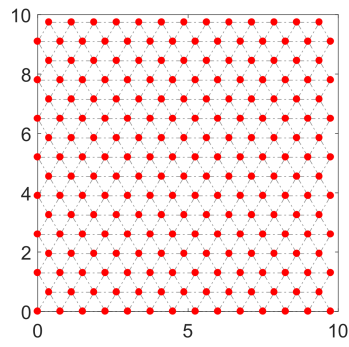

(j) Top-10: 218 agents

Figure 4: Illustration of the 10 different network topologies in the square range with $L=10 r_{c}$ and density $\rho \approx 2 / r_{c}^{2}$.

\subsection{Analyze $\alpha$ under General Data}

In order to reveal the general rules, ten topological structures are used in this subsection, which are plotted in Figure 4. The topologies in the square range with $L=10 r_{c}$ are described as follows.

- Top-1: Random place $n=\rho \times L^{2}$ agents in a circular area of radius $\frac{L}{2}$. As shown in Figure 4(a), a total of 200 agents are generated.

- Top-2: A squared area with $L=10 r_{c}$ is divided into $\frac{L^{2}}{r_{c}^{2}}$ small squares on average, and $\rho$ agents are randomly generated in each square. As shown in Figure 4(b), a total of 200 agents are generated. 


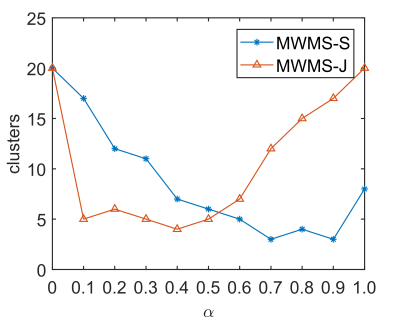

(a) Top-1

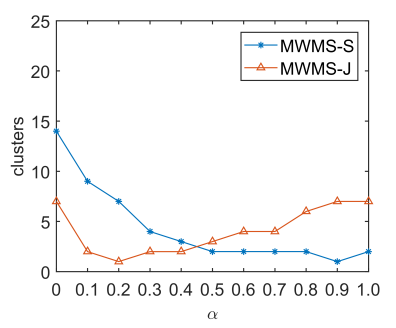

(e) Top-5

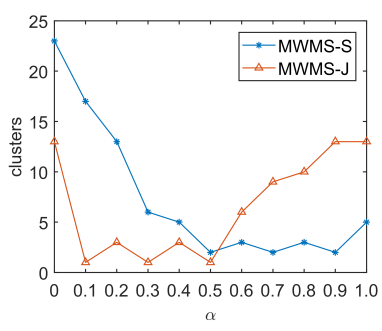

(b) Top-2

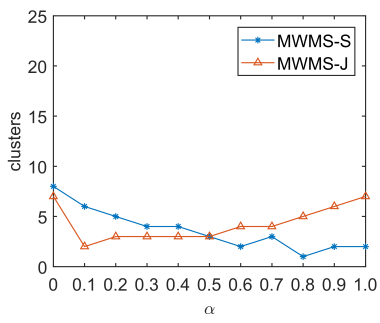

(f) Top-6

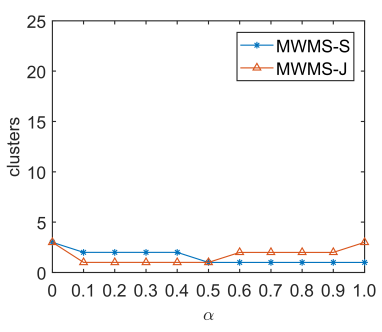

(c) Top-3

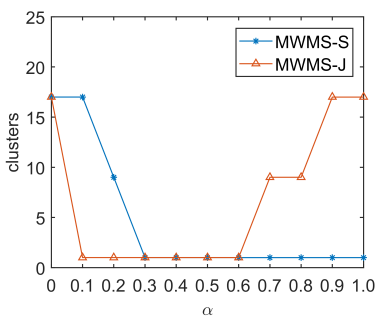

(g) Top-7

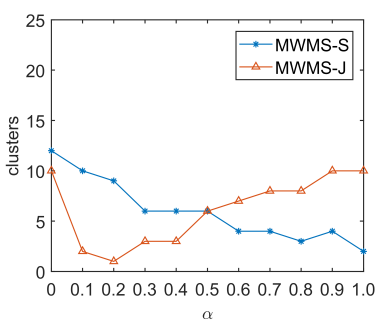

(d) Top-4

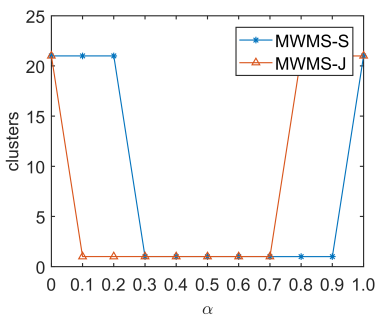

(h) Top-8

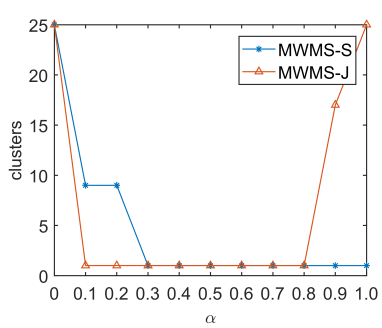

(i) Top-9

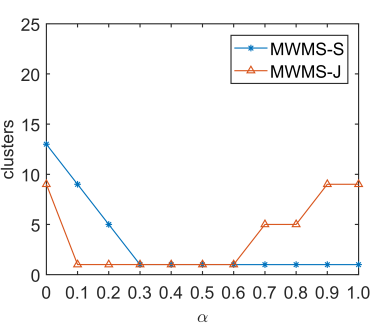

(j) Top-10

Figure 5: Parameter analysis of $\alpha$ : the evaluation measures is number of clusters.

- Top-3: $n=\frac{1}{2} \rho L^{2}$ agents are randomly generated in two circles with $\left(\frac{L}{2+2 \sqrt{2}}, \frac{L}{2+2 \sqrt{2}}\right)$, $\left(-\frac{L}{2+2 \sqrt{2}},-\frac{L}{2+2 \sqrt{2}}\right)$ as the center and $\frac{L}{2+\sqrt{2}}$ as the radius respectively. As shown in Figure $4(\mathrm{c})$, a total of 200 agents are generated.

- Top-4: Random place $n=\rho \times L^{2}$ agents in a range consisting of a circle with radius $R_{\text {Outer }}=\frac{L}{2}$ and a circle with radius $R_{\text {Inner }}=0.6 R_{\text {Outer }}$. As shown in Figure $4(\mathrm{~d})$, a total of 200 agents are generated.

- Top-5: In an isosceles triangle with $L$ as the base and height, $n=\rho \times L^{2}$ agents are randomly generated. As shown in Figure 4(e), a total of 200 agents are generated.

- Top-6: Divide a square with $L$ equally into 9 squares with side length $l=\frac{L}{3}$. The four squares at the right angle are removed and $n=\rho \times L^{2}$ agents are generated randomly in the remaining five squares. As shown in Figure 4(f), a total of 200 agents are generated.

- Top-7: It is a neat topology generated by dividing a circle with a radius of $r=\frac{L}{2}$ into $m=\frac{r}{0.5 r_{c}}$ equal layers and placing an agent at equal radian intervals $\kappa r_{c}, \kappa$ is 
Table 1: The statistics of 5 topologies in the same range $\left(L=10 r_{c}\right)$ and with varying densities $\rho$.

\begin{tabular}{|l|c|c|c|c|c|}
\hline No. & $\rho$ & \#Agents & \#A-edges & \#M-edges & \#Tri \\
\hline \hline D-1 & $4 / r_{c}^{2}$ & 400 & 2100 & 2100 & 3642 \\
\hline D-2 & $8 / r_{c}^{2}$ & 800 & 8778 & 8778 & 35996 \\
\hline D-3 & $12 / r_{c}^{2}$ & 1200 & 20314 & 20314 & 133541 \\
\hline D-4 & $16 / r_{c}^{2}$ & 1600 & 36110 & 36110 & 320667 \\
\hline D-5 & $20 / r_{c}^{2}$ & 2000 & 56647 & 56647 & 637085 \\
\hline
\end{tabular}

Table 2: The statistics of 5 topologies in the same density $\left(4 / r_{c}^{2}\right)$ and with varying $L$.

\begin{tabular}{|l|c|c|c|c|c|}
\hline No. & $L$ & \#Agents & \#A-edges & \#M-edges & \#Tri \\
\hline \hline L-1 & $5 r_{c}$ & 100 & 480 & 480 & 816 \\
\hline L-2 & $10 r_{c}$ & 400 & 2072 & 2072 & 3532 \\
\hline L-3 & $15 r_{c}$ & 900 & 4883 & 4883 & 8635 \\
\hline L-4 & $20 r_{c}$ & 1600 & 8449 & 8448 & 15834 \\
\hline L-5 & $25 r_{c}$ & 2500 & 13927 & 13927 & 24889 \\
\hline
\end{tabular}

related to the density $\rho$. As shown in Figure $4(\mathrm{~g})$ with $\kappa=0.8$, a total of 221 agents are generated.

- Top-8: It is a neat topology generated by dividing $\pi$ into 25 equal parts, placing an agent at equal distances $d=0.5 r_{c}$ in each direction. As shown in Figure 4(h), a total of 217 agents are generated.

- Top-9: Neatly place about $\left\lceil\sqrt{\rho L^{2}}\right\rceil^{2}$ agents in a square area with a side length of $L$. As shown in Figure 4(i), a total of 225 agents are generated.

- Top-10: Different from Top-9. From the bottom up, Top-10 shifts each row a little bit to the left. It's worth noting that each triangle is an equilateral triangle. As shown in Figure 4(j), a total of 218 agents are generated.

The effect of the trade-off parameter, i.e., $\alpha$ in Eq. 9, on the consensus of MAS is shown in Figure 5. As can be seen, the performance of consensus (measured by number of eventually formed clusters) vary slightly as $\alpha$ changes. The most common phenomenon for all of these figures is that they display a trend of first increase and then drop. However, the "knee points" may be a little bit different for different datasets. On the whole, as $\alpha$ approaches a certain value that are larger than 0.5 but less than 1.0 for MWMS-S and larger than 0 but less than 0.5 for MWMS-J, the best performance can be obtained. When $\alpha$ is set to be exactly 0 , which means that the proposed method degenerates into the basic consensus method, the values of the evaluation measures are decreased.

The results have confirmed the necessity of utilizing the motif-based higher-order structure and the effectiveness of the proposed method. However, since $\alpha$ plays a role of balancing the influence of quantity of the connections in view of the number of neighbors and quality 
Table 3: Results of the number of clusters that converge with different $\alpha$ based on MWMS-S.

\begin{tabular}{|l|c|c|c|c|c|c|c|c|c|c|c|}
\hline No. & $\mathbf{0}$ & $\mathbf{0 . 1}$ & $\mathbf{0 . 2}$ & $\mathbf{0 . 3}$ & $\mathbf{0 . 4}$ & $\mathbf{0 . 5}$ & $\mathbf{0 . 6}$ & $\mathbf{0 . 7}$ & $\mathbf{0 . 8}$ & $\mathbf{0 . 9}$ & $\mathbf{1 . 0}$ \\
\hline \hline D-1 & 20 & 17 & 12 & 11 & 7 & 6 & 5 & 3 & 4 & 3 & 8 \\
\hline D-2 & 23 & 14 & 8 & 6 & 4 & 1 & 1 & 1 & 1 & 1 & 1 \\
\hline D-3 & 22 & 13 & 9 & 5 & 4 & 3 & 2 & 1 & 2 & 3 & 1 \\
\hline D-4 & 26 & 15 & 12 & 11 & 9 & 12 & 11 & 7 & 6 & 6 & 5 \\
\hline D-5 & 27 & 11 & 6 & 5 & 5 & 1 & 3 & 3 & 3 & 3 & 4 \\
\hline \hline L-1 & 5 & 3 & 2 & 1 & 1 & 1 & 1 & 1 & 1 & 1 & 1 \\
\hline L-2 & 20 & 15 & 12 & 9 & 5 & 2 & 2 & 2 & 2 & 2 & 3 \\
\hline L-3 & 52 & 33 & 27 & 18 & 14 & 13 & 8 & 3 & 2 & 4 & 2 \\
\hline L-4 & 95 & 67 & 47 & 39 & 30 & 28 & 18 & 14 & 5 & 4 & 4 \\
\hline L-5 & 147 & 104 & 80 & 64 & 47 & 46 & 29 & 18 & 19 & 17 & 16 \\
\hline
\end{tabular}

Table 4: Results of the number of clusters that converge with different $\alpha$ based on MWMS-J.

\begin{tabular}{|l|c|c|c|c|c|c|c|c|c|c|c|}
\hline No. & $\mathbf{0}$ & $\mathbf{0 . 1}$ & $\mathbf{0 . 2}$ & $\mathbf{0 . 3}$ & $\mathbf{0 . 4}$ & $\mathbf{0 . 5}$ & $\mathbf{0 . 6}$ & $\mathbf{0 . 7}$ & $\mathbf{0 . 8}$ & $\mathbf{0 . 9}$ & $\mathbf{1 . 0}$ \\
\hline \hline D-1 & 20 & 5 & 6 & 5 & 4 & 5 & 7 & 12 & 15 & 17 & 20 \\
\hline D-2 & 18 & 4 & 3 & 2 & 3 & 4 & 6 & 6 & 10 & 15 & 18 \\
\hline D-3 & 17 & 3 & 4 & 5 & 4 & 5 & 5 & 8 & 10 & 11 & 17 \\
\hline D-4 & 16 & 4 & 6 & 6 & 7 & 8 & 9 & 8 & 8 & 10 & 16 \\
\hline D-5 & 15 & 4 & 5 & 4 & 6 & 5 & 7 & 5 & 7 & 8 & 15 \\
\hline \hline L-1 & 4 & 1 & 1 & 1 & 1 & 1 & 1 & 1 & 3 & 2 & 4 \\
\hline L-2 & 19 & 2 & 4 & 3 & 2 & 4 & 7 & 7 & 10 & 14 & 19 \\
\hline L-3 & 38 & 6 & 6 & 8 & 13 & 11 & 13 & 19 & 26 & 31 & 38 \\
\hline L-4 & 76 & 12 & 13 & 12 & 16 & 19 & 28 & 30 & 46 & 55 & 76 \\
\hline L-5 & 117 & 21 & 20 & 24 & 26 & 29 & 38 & 55 & 74 & 85 & 117 \\
\hline
\end{tabular}

of the connections based on the motif structure, the optimal value may be different for different datasets. In addition, as shown in Top- 8 with a chain structure, if only higherorder structures are considered without lower-order structures, that is, $\alpha=1$, the neighbor structure becomes fragile, thus the consensus process of MAS can be easily affected. Actually, the addition of the motif matrix $M$ makes each agent more constrained, and more constraints would make the propagation more stable, which is coincident to our intuition and accounts for what have been shown in Figure 5.

\subsection{Enhancement of Experimental Data}

In order to explore the larger scale multi-agent system, the topologies in this subsection are described in (Vicsek, Czirók, Ben-Jacob, Cohen, \& Shochet, 1995) whose simulation are carried out in a square shaped of linear size $L$ and with density $\rho=n / L^{2}$. 
Firstly, we place the agent in the square area of $L=10 r_{c}$. In order to ensure the connectivity of network topology, we divided the square area into several $0.5 r_{c} \times 0.5 r_{c}$ small squares. The number of agents in each $0.5 r_{c} \times 0.5 r_{c}$ small square is $1,2,3,4$ and 5 respectively. In other words, the density is $4 / r_{c}^{2}, 8 / r_{c}^{2}, 12 / r_{c}^{2}, 16 / r_{c}^{2}, 20 / r_{c}^{2}$, respectively. The statistic of these 5 topologies is shown in Table 1, which contains the number of agents (i.e. \#Agents), the number of edges of adjacency matrix (i.e. \#A-edges), the number of edges of motif matrix (\#M-edges) and the number of triangle motif instances (i.e. \#Tri). Secondly, agents are distributed in five different ranges of $L=5 r_{c}, 10 r_{c}, 15 r_{c}, 20 r_{c}, 25 r_{c}$ with the same $\rho=4 / r_{c}^{2}$. The statistic of these 5 topologies is shown in Table 2 . From the two tables, we can see that the number of edges of each network topology is not the same, and the proportion of triangle motif also differs significantly from one to another.

In this experiment, we test the values of $\alpha \in\{0,0.1,0.2,0.3,0.4,0.5,0.6,0.7,0.8,0.9,1\}$ and report the results in terms of the cluster number in Table 3 and Table 4 . We can see that the best value of $\alpha$ varies from one topology to another. It is confirmed that in each of the topologies, the proposed methods outperform the existing lower-order structure based methods.

Furthermore, the two tables are firstly analyzed horizontally. Under the same topology, we can observe that the number of clusters that eventually converge in the multi-agent system has a decreasing trend with the increase of $\alpha$, which means that in the case of sufficient network topology density, a higher value of $\alpha$ can promote the consensus of the system. However, when $\alpha$ reaches a certain size, the results are relatively stable, without too many large fluctuations. We can conclude that MWMS is relatively stable and insensitive to $\alpha$. As long as the higher-order structure is considered, the system performance can be effectively improved.

Then, from the longitudinal analysis under the same $\alpha$, from D-1 to D-5, multi-agent systems with lower density tend to have better convergence performance, especially after $\alpha$ value is greater than 1 . It can be concluded that, when the magnitude of the trade-off of motif matrix is the same or even greater than that of the adjacency matrix, the lower density topological structure has a better effect. The main reason is that the motif matrix has some triangle motifs in it that we should ignore. Within the same range, the number of triangle motifs of low-density topologies is less, whereas the magnitude of triangle motifs of high-density topologies is much higher, and there must be some triangle motifs in a large number of triangle motifs that hinder the convergence of the system. Therefore, we hypothesized that it would be better to simplify its information again in the motif matrix. From L-1 to L-5, the convergence performance of the system will decline if the range is increased with a certain density. The reason is that, in the large network topology, the agent is more likely to fall into the local optimal convergence position, which leads to the system splitting.

As for how to select an appropriate $\alpha$ for an unknown network where the ground truth is not accessible, we can make use of the structure measure, e.g., characteristics, density and range of initial network, proportion of triangle motif, etc. We can draw a conclusion that the convergence performance and generality of MWMS considering motif matrix proposed in this paper is more advantageous than that of traditional multi-agent system, which explores deeper relationships among agents and makes the agent network more stable. 


\section{Conclusion}

In this paper, we for the first time propose a motif-aware weighted multi-agent system for enhancing consensus, which can shed light on the higher-order structure of the network by motif mining. In addition, by seamlessly integrating the motif-based higher-order features with the edge-based lower-order structure of the network, i.e., hybrid-order structure, we consider not only the quantity but also the quality of connections. Two baseline methods are used for MWMS. What's more, compared with these two traditional consensus protocols, our methods are more robust. The hundred of random network experiments demonstrate that the proposed method achieves better performance over the baseline algorithms.

\section{Acknowledgments}

This work was supported in part by the Natural Science Foundation of China (NSFC) under Grant 62006047, 61976052 and 61876043. Guangqiang Xie and Yang Li (corresponding author) are working at the School of Computers of Guangdong University of Technology. We also would like to thank the JAIR reviewers and editor for their insightful and helpful suggestions.

\section{References}

Amato, C. (2018). Decision-making under uncertainty in multi-agent and multi-robot systems: Planning and learning. In IJCAI, pp. 5662-5666.

Bhargava, N. (2019). Multi-agent coordination under uncertain communication. In $A A A I$, pp. $9878-9879$.

Bloembergen, D., Tuyls, K., Hennes, D., \& Kaisers, M. (2015). Evolutionary dynamics of multi-agent learning: A survey. J. Artif. Intell. Res., 53, 659-697.

Bond, A. H. (1988). An analysis of problems and research in DAI. Readings in distributed artificial intelligence, 3-35.

Cao, Y., \& Ren, W. (2009). Optimal linear-consensus algorithms: An lqr perspective. IEEE Transactions on Systems, Man, and Cybernetics, Part B (Cybernetics), 40(3), 819-830.

Cao, Y., Ren, W., Casbeer, D. W., \& Schumacher, C. (2016). Finite-time connectivitypreserving consensus of networked nonlinear agents with unknown lipschitz terms. IEEE Trans. Automat. Contr., 61(6), 1700-1705.

Coletto, M., Garimella, K., Gionis, A., \& Lucchese, C. (2017). A motif-based approach for identifying controversy. In ICWSM, pp. 496-499.

da Silva, R. R., Wu, B., Dai, J., \& Lin, H. (2016). Extended abstract: Formal design of cooperative multi-agent systems. In $A A A I$.

Das, M., Dhami, D. S., Kunapuli, G., Kersting, K., \& Natarajan, S. (2019). Fast relational probabilistic inference and learning: Approximate counting via hypergraphs. In $A A A I$, pp. 7816-7824.

Dobbe, R., Fridovich-Keil, D., \& Tomlin, C. (2017). Fully decentralized policies for multiagent systems: An information theoretic approach. In NIPS, pp. 2945-2954. 
Dong, W. (2010). Distributed optimal control of multiple systems. Int. J. Control, 83(10), $2067-2079$.

Dutta, P. S., Jennings, N. R., \& Moreau, L. (2005). Cooperative information sharing to improve distributed learning in multi-agent systems. J. Artif. Intell. Res., 24, 407-463.

Ephrati, E., \& Rosenschein, J. S. (1996). Deriving consensus in multiagent systems. Artif. Intell., $87(1-2), 21-74$.

Han, Y., \& Gmytrasiewicz, P. J. (2018). Learning others' intentional models in multi-agent settings using interactive pomdps. In NeulPS, pp. 5639-5647.

Huang, L., Wang, C., \& Chao, H. (2018). A harmonic motif modularity approach for multi-layer network community detection. In ICDM, pp. 1043-1048.

Huang, L., Wang, C., \& Chao, H. (2019). Higher-order multi-layer community detection. In AAAI, pp. 9945-9946.

Jadbabaie, A., Lin, J., \& Morse, A. S. (2003). Coordination of groups of mobile autonomous agents using nearest neighbor rules. IEEE Trans. Automat. Contr., 48(6), 988-1001.

Jiang, J., \& Lu, Z. (2018). Learning attentional communication for multi-agent cooperation. In NeulPS, pp. 7265-7275.

Kashani, Z. R. M., Ahrabian, H., Elahi, E., Nowzari-Dalini, A., Ansari, E. S., Asadi, S., Mohammadi, S., Schreiber, F., \& Masoudi-Nejad, A. (2009). Kavosh: a new algorithm for finding network motifs. BMC Bioinformatics, 10, 318.

Kim, J. K., \& Choi, S. (2009). Clustering sequence sets for motif discovery. In NIPS, pp. 970-978.

Kraus, S. (1997). Negotiation and cooperation in multi-agent environments. Artif. Intell., 94(1-2), 79-97.

Le, D., \& Plaku, E. (2018). Cooperative, dynamics-based, and abstraction-guided multi-robot motion planning. J. Artif. Intell. Res., 63, 361-390.

Le, D., \& Plaku, E. (2019). Multi-robot motion planning with dynamics via coordinated sampling-based expansion guided by multi-agent search. IEEE Robotics and Automation Letters, 4 (2), 1868-1875.

Li, M., Yang, W., Cai, Z., Yang, S., \& Wang, J. (2019). Integrating decision sharing with prediction in decentralized planning for multi-agent coordination under uncertainty. In $I J C A I$, pp. $450-456$.

Li, P., Dau, H., Puleo, G. J., \& Milenkovic, O. (2017). Motif clustering and overlapping clustering for social network analysis. In 2017 IEEE Conference on Computer Communications, INFOCOM 2017, Atlanta, GA, USA, May 1-4, 2017, pp. 1-9.

Li, P., Cai, Y., Wang, C., Liang, M., \& Zheng, Y. (2019). Higher-order brain network analysis for auditory disease. Neural Processing Letters, 49(3), 879-897.

Li, P., Huang, L., Wang, C., Huang, D., \& Lai, J. (2018). Community detection using attribute homogenous motif. IEEE Access, 6, 47707-47716. 
Li, P., Huang, L., Wang, C., \& Lai, J. (2019). Edmot: An edge enhancement approach for motif-aware community detection. In Proceedings of the 25th ACM SIGKDD International Conference on Knowledge Discovery \& Data Mining, KDD 2019, Anchorage, AK, USA, August 4-8, 2019., pp. 479-487.

Lin, W., Xiao, X., Xie, X., \& Li, X. (2017). Network motif discovery: A GPU approach. IEEE Trans. Knowl. Data Eng., 29(3), 513-528.

Lin, X., Adams, S. C., \& Beling, P. A. (2019). Multi-agent inverse reinforcement learning for certain general-sum stochastic games. J. Artif. Intell. Res., 66, 473-502.

Ma, J., Zheng, Y., \& Wang, L. (2015). Lqr-based optimal topology of leader-following consensus. International Journal of Robust and Nonlinear Control, 25(17), 3404-3421.

Milo, R., Shen-Orr, S., Itzkovitz, S., Kashtan, N., Chklovskii, D., \& Alon, U. (2002). Network motifs: Simple building blocks of complex networks. Science, 298(5594), 824-827.

Motsch, S., \& Tadmor, E. (2014). Heterophilious dynamics enhances consensus. SIAM Review, 56(4), 577-621.

Nebel, B., Bolander, T., Engesser, T., \& Mattmüller, R. (2019). Implicitly coordinated multiagent path finding under destination uncertainty: Success guarantees and computational complexity. J. Artif. Intell. Res., 64, 497-527.

Newman, M. E. (2001). The structure of scientific collaboration networks. Proceedings of the national academy of sciences, 98(2), 404-409.

Newman, M. E. (2006). Modularity and community structure in networks. Proceedings of the national academy of sciences, 103(23), 8577-8582.

Newman, M. E., \& Park, J. (2003). Why social networks are different from other types of networks. Physical review E, 68(3), 036122.

Olfati-Saber, R. (2005). Ultrafast consensus in small-world networks. In Proceedings of the 2005, American Control Conference, 2005., pp. 2371-2378. IEEE.

Olfati-Saber, R., Fax, J. A., \& Murray, R. M. (2007). Consensus and cooperation in networked multi-agent systems. Proc. IEEE, 95(1), 215-233.

Ren, W., \& Beard, R. W. (2005). Consensus seeking in multiagent systems under dynamically changing interaction topologies. IEEE Trans. Automat. Contr., 50(5), 655-661.

Song, J., Ren, H., Sadigh, D., \& Ermon, S. (2018). Multi-agent generative adversarial imitation learning. In NeulPS, pp. 7472-7483.

Song, S., Zou, Z., \& Liu, K. (2016). Triangle-based representative possible worlds of uncertain graphs. In Database Systems for Advanced Applications - 21st International Conference, DASFAA 2016, Dallas, TX, USA, April 16-19, 2016, Proceedings, Part II, pp. 283-298.

Vicsek, T., Czirók, A., Ben-Jacob, E., Cohen, I., \& Shochet, O. (1995). Novel type of phase transition in a system of self-driven particles. Physical review letters, 75 (6), 1226.

Wai, H., Yang, Z., Wang, Z., \& Hong, M. (2018). Multi-agent reinforcement learning via double averaging primal-dual optimization. In NeulPS, pp. 9672-9683. 
Wang, S., Wei, Z., Fan, Z., Liu, Y., \& Huang, X. (2019). A multi-agent communication framework for question-worthy phrase extraction and question generation. In $A A A I$, pp. $7168-7175$.

Wen, Y., Gao, L., Fu, H., Zhang, F., \& Xia, S. (2019). Graph CNNs with motif and variable temporal block for skeleton-based action recognition. In $A A A I$, pp. 8989-8996.

Wernicke, S., \& Rasche, F. (2006). FANMOD: A tool for fast network motif detection. Bioinformatics, 22(9), 1152-1153.

Xu, B., \& He, W. (2018). Event-triggered cluster consensus of leader-following linear multi-agent systems. J. Artif. Intell. Soft Comput. Res., 8(4), 293-302.

Zhang, F., Wang, W., \& Zhang, H. (2015). Design and analysis of distributed optimal controller for identical multiagent systems. Asian Journal of Control, 17(1), 263-273.

Zheng, Y., Ma, J., \& Wang, L. (2018). Consensus of hybrid multi-agent systems. IEEE Trans. Neural Netw. Learning Syst., 29(4), 1359-1365.

Zhou, D., Zhang, S., Yildirim, M. Y., Alcorn, S., Tong, H., Davulcu, H., \& He, J. (2017). A local algorithm for structure-preserving graph cut. In $K D D$, pp. 655-664. 